
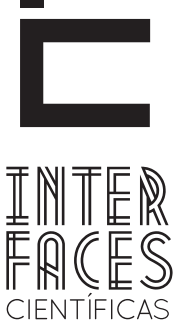

SAÚDE E AMBIENTE

ISSN IMPRESSO 2316-3313

ISSN ELETRÔNICO 2316-3798

\title{
GRAVIDEZ ABDOMINAL COM RECÉM-NASCIDO VIVO: RELATO DE CASO
}

Max Oliveira Menezes ${ }^{1}$

Maria Inês Brandão Bocardi ${ }^{3}$
Clarissa Melo Menezes²

foi remanejado para a Unidade de Cuidado Intermediário Neonatal Convencional. Com relação à puérpera, foi optado por não remover a placenta durante a abordagem e posterior transferência para Unidade de Terapia Intensiva de um Hospital Geral. Mãe e bebê receberam alta em boas condições de saúde

\section{PALAVRAS-CHAVE}

Gravidez Abdominal. Gravidez Ectópica. Vitalidade Fetal. 


\section{ABSTRACT}

Abdominal pregnancy constitutes a rare fact in obstetrics, becoming even rarer event when it is an abdominal pregnancy that progressed to live and viable fetus until birth. In this study, we report a case of abdominal pregnancy in a primigravida 24 , no diagnosis during prenatal care. How conduct was performed surgical procedure for fetal extraction with spinal anesthesia assistance. Live fetus, single, female, weighing 2,220 g. Presented Apgar score in the first minute and 7 at the fifth minute 9. Implemented under oxygen hood.

\section{RESUMEN}

El embarazo abdominal constituye un evento raro en la obstetricia, convirtiéndose en caso aún más raro cuando se trata de un embarazo abdominal que ha progresado con feto vivo y viable hasta el nacimiento. En este estudio, los autores informan de un caso de embarazo abdominal en una primigesta de 24 años y sin diagnóstico durante la atención prenatal. Cómo llevar a cabo procedimiento quirúrgico se realizó para la extracción fetal con la ayuda de la anestesia espinal. Feto vivo, único, femenino, con un peso de 2.220 g. Presentado Apgar Score en el primer minuto y 7 a los cinco minutos 9 . Implementadas bajo campana de oxígeno. El recién nacido fue reasignada a la Unidad
The newborn was reassigned to Care Unit Neonatal Intermediate Conventional. Regarding postpartum, was chosen not remove the placenta during the approach and subsequent transfer to the intensive care unit of a general hospital. Mother and baby were discharged in good health.

\section{KEYWORDS}

Abdominal Pregnancy. Ectopic Pregnancy. Fetal Vitality.

Neonatal de Cuidados Intermedios convencional. En cuanto posparto, fue elegido no elimina la placenta durante la aproximación y posterior traslado a la Unidad de Cuidados Intensivos de un Hospital General. La madre y el bebé fueron dados de alta en buen estado de salud.

\section{PALABRAS CLAVE}

Embarazo abdominal. Embarazo ectópico. Vitalidad fetal. 


\section{INTRODUCÃO}

A gravidez ectópica (GE) é caracterizada pela implantação ovular fora da cavidade uterina, ou seja, na trompa (97\%), no ovário (0,5\%), no peritônio (gravidez abdominal, 1 a 1,4\%), cervical (0,1\%), intramural $(0,6 \%)$. Podem ser observados vários fatores de risco para o desenvolvimento de uma GE como o uso de antibióticos para tratamento da doença inflamatória pélvica (DIP), raça negra, gestações tardias, cirurgias reparadoras, técnicas de reprodução assistida, métodos contraceptivos como o dispositivo intrauterino (DIU), promiscuidade, endometriose, anomalias uterinas e gravidez ectópica prévia (GONZALES, 2002; YUSUF, et al., 2010; ALMEIDA, et al., 2008).

Nos últimos anos, atingiu a proporção de 1 em cada 100 gravidezes normais nos Estados Unidos da América, com taxa de mortalidade de 3,8 para 10.000 casos (SOARES, 2002; CHAVES NETO, 2005). No Brasil não existem medidas de prevalência nacionais ou mesmo regionais dessa patologia (SILVA FILHO; MARQUES; NUNES, 2013; BRITO, 2013).

Especificamente na gravidez ectópica abdominal os índices atingem variabilidade de 0,5 a 0,66 \% dos casos (NEME, 2005). De acordo com dados de Santos e outros autores (1999), existe a proporção de 1 para 10.000 a 1 para 64.000 nascimentos de crianças decorrentes de gravidez ectópica abdominal. A sobrevivência neonatal é de cerca de $20 \%$ nas maiores séries publicadas.

Neme (2005) e Chaves Neto (2005) classificam a gravidez abdominal em primária e secundária. A primária ocorre quando há nidação direta sobre a serosa peritoneal e é uma forma de difícil comprovação. Já a secundária ocorre primeiro a implantação do ovo na trompa ou ovário, seguido de abortamento para a cavidade abdominal e, posterior reimplantação do saco gestacional, preferentemente no ligamento largo, no fundo de saco de Douglas ou no omento.
O diagnóstico é realizado por sinais clínicos, dosagem plasmática da fração beta do hormônio gonadotrofina coriônica (ß-HCG) e ultrassonografia transvaginal. Dentre os sinais clínicos, podemos citar: dor abdominal agravada pelos movimentos fetais; náuseas; vômito; alterações do trânsito intestinal; partes fetais facilmente palpáveis; dificuldade e dor nas tentativas de mobilização fetal; ausência de resposta contrátil uterina após administração de ocitócito (Sinal de Braxton-Hicks) e ao toque vaginal o colo apresenta-se muito elevado ou retropúdico (NEME, 2005).

As principais complicações antenatais incluem dor abdominal, ruptura do saco gestacional com hemorragia para a cavidade peritoneal, sangramento vaginal, apresentação anômala, insuficiência placentária e óbito fetal (HOLZHACKER, 2008).

No que diz respeito às formas de tratamento, a conduta dependerá da idade e vitalidade fetal. Se o feto estiver vivo, pode-se optar por atitude conservadora, aguardando a maturidade fetal. Nesse caso, pratica-se a laparotomia e o momento crucial dessa intervenção é a extração da placenta, pois o descolamento da mesma pode provocar lesões de órgãos ou estruturas, e/ou hemorragia de difícil controle (ALMEIDA, et al., 2008).

O prognóstico materno depende de fatores como diagnóstico precoce, a necessidade de transfusão sanguínea, qualidade do hospital e atendimento médico. Os óbitos são principalmente causados por hemorragia e infecção. 0 risco de morte materna é 7,7 vezes superior ao da prenhez tubária e 90 vezes mais elevado do que na gestação uterina (REZENDE; MONTENEGRO, 2006; CHAVES NETO, 2005).

Este estudo teve como objetivo relatar um caso raro de gestação ectópica abdominal que progrediu com feto viável sem malformações e que não foi 
diagnosticado durante o pré-natal nas ultrassonografias realizadas. Entende-se que por se tratar de um fato ainda raro, todos os casos devem ser descritos e relatados viabilizando a discussão no âmbito acadêmico e profissional.

\section{RELATO DE CASO}

Paciente de 24 anos, primigesta, deu entrada no dia 20/5/2014 em uma Maternidade de Alto Risco na cidade de Aracaju/Se, com DUM de 20/10/2013, apresentando dor em baixo ventre há 6 dias, negando perda de líquido amniótico ou sangue via vaginal, movimentação fetal positiva. De acordo com exames realizados no pré-natal:

-14/11/13:

Beta - HCG: reagente.

- 22/11/13:

Hb: 12,5 g/dl; Ht: 33,9\%; G:71 mg/dl; PLT: 278; VDRL: não reagente; $A B O / R H: A+$; Sumário de urina: piócitos 4/5; AntiHCV: não reagente; HIV: não regente.

- 23/11/13:

Ultrassonografia obstétrica: útero com saco gestacional de contornos regulares, implantação corporal contendo embrião único. Gestação tópica de 7 semanas. Tempo de amenorreia ignorado. Vitalidade fetal preservada.

- 26/11/13:

Citomegalovírus IgG reagente (> $250 \mathrm{UA} / \mathrm{ml}$ ); rubéola IgG reagente $(84,1 \mathrm{UI} / \mathrm{ml}) ; \mathrm{HBsAg}$ não reagente. - 3/2/14:

Ultrassonografia obstétrica: útero aumentado de volume contendo em seu interior feto único, longitudinal, apresentação e dorso variável. Placenta com inserção fúndica, textura homogênea (Grau 0). Gestação tópica de 18 semanas. Vitalidade fetal preservada.

- 26/3/14:

Ultrassonografia morfológica: feto único, vivo com idade gestacional estimada em 25 semanas e 2 dias; concordante com idade gestacional menstrual; es- tudo morfológico sem alterações para idade gestacional; peso fetal estimado em $885 \mathrm{~g}$; crescimento fetal adequado; placenta prévia centro total.

Ao exame médico obstétrico pode-se observar: 33 cm de altura uterina, colo uterino fechado, apresentação fetal transversa, batimentos cardíacos fetais (BCF) de 146 bpm, posição lateral direita.

Em sua admissão foi realizada a solicitação de uma ultrassonografia que apresentou os seguintes achados ecográficos: Placenta anterior lateral esquerda de inserção baixa (ocluindo o OCT do colo), líquido amniótico diminuído, feto único, situação transversa com pólo cefálico à esquerda, apresentação fetal córmica. Exame conclusivo de placenta prévia total (PP).

Diante da IG de 34 semanas (pela USG de $3 / 2 / 2014$ ) e hipótese diagnóstica de placenta prévia total, a gestante foi admitida na maternidade para realização de corticoterapia com o objetivo de amadurecimento pulmonar fetal. Nesta ocasião também foi prescrito antiespasmódico pelo médico plantonista.

Complementando a rotina admissional, foi solicitado exame de fator sanguíneo, hemograma, teste rápido para HIV (resultado negativo). Como medida preventiva foi solicitada duas unidades de concentrados de hemácias.

No dia 22/5/2014 a gestante foi encaminhada ao Centro Obstétrico para a realização parto cesariano. Foi admitida em sala cirúrgica e implementado procedimentos de rotina, como: monitorização (PAS: $150 \mathrm{x}$ $96 \mathrm{mmHg}$, FC: 101 bpm, Sat: 98\%), sondagem vesical de demora, raquianestesia (utilizado morfina e neocaína pesada). Durante o transoperatório, foi necessária a realização de anestesia geral balanceada com subsequente intubação endotraqueal.

Após a realização da diérese, detectou-se uma inserção placentária e fetal totalmente abdominal (à esquerda). Após a retirada do feto, notou-se um útero 
de pequenas dimensões e a placenta estava aderida ao fundo de útero e epiplon.

Figura 1- A: Abertura da cavidade abdominal visualizando útero fechado;

B: implantação do cordão umbilical na cavidade abdominal

A

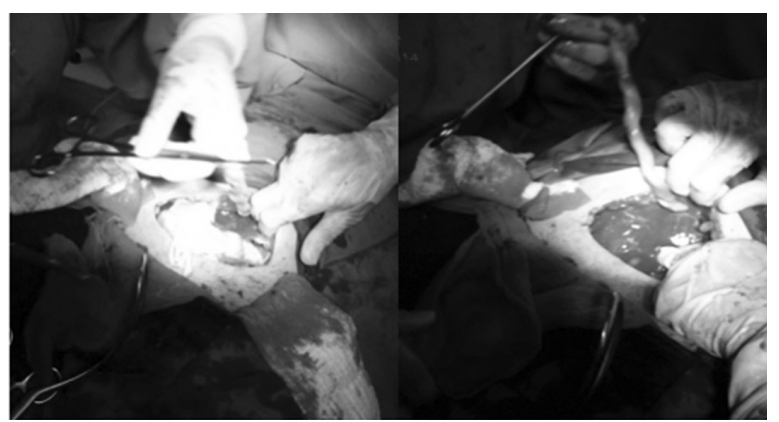

Fonte: Dados da pesquisa, 2014.

Após 30 minutos da extração fetal a pressão arterial sistólica era de 116 e a diastólica de $53 \mathrm{mmHg}$, foi puncionado um segundo acesso venoso periférico e iniciado a hemotransfusão ( $1^{\mathrm{a}}$ unidade de concentrado de hemácias).

A equipe médica optou por não remover a placenta, em virtude de uma inserção irregular abrangendo várias estruturas abdominais. Na tentativa de conter possíveis focos hemorrágicos, utilizaram-se esponjas hemostáticas.

Após a síntese, foi realizada uma USG para descartar a possibilidade de acumulo de líquido na cavidade (sangue). Evidenciado que não houve persistência de pontos hemorrágicos ativos.

A puérpera permaneceu monitorizada na sala cirúrgica e em uso de ventilação mecânica. Após reavaliação do quadro clínico, optou-se em realizar a extubação e oferta de 02 por meio de cateter tipo óculos. Paralela- mente, foi solicitada vaga em unidade de terapia intensiva (UTI) da rede de assistência à saúde estadual.

Durante o procedimento cirúrgico as medicações usadas pelo anestesiologista foram: cefalotina $2 \mathrm{~g}$; decadron 10mg; nausedron 8mg; efedrina $25 \mathrm{mg} \mathrm{(2}$ ampolas); oxitocina 10UI; profenid 100mg; dipirona $2 \mathrm{~g}$; etomidato $5 \mathrm{mg}$; fentanil $150 \mathrm{mg}$; midazolam, nilperidol, atropina $1 \mathrm{mg}$ e neostigmina.

No pós-operatório imediato foi prescrito pelos obstetras: hidratação com ringer lactato e soro glicosado a $5 \%$ (2000 $\mathrm{ml}$ ), cefalotina IV de $6 / 6 \mathrm{~h}$, dipirona IV de $6 / 6 \mathrm{~h}$, profenid IV de 12/12 h, nausedron IV de 8/8 h, 2 unidades de concentrado de hemácias e 2 unidades de plasma.

0 recém-nascido foi recepcionado pela equipe de neonatologia e realizado cuidados de rotina. Pesou $2220 \mathrm{~g}$, obteve índice de Apgar de 7 no primeiro minuto e 9 no quinto minuto. Manteve-se observação em berço aquecido e oferta de 02 por meio de hood. Posteriormente foi encaminhado para assistência na unidade de cuidados intermediários neonatal convencional.

Figura 2 - RN do sexo feminino (gestação abdominal total)

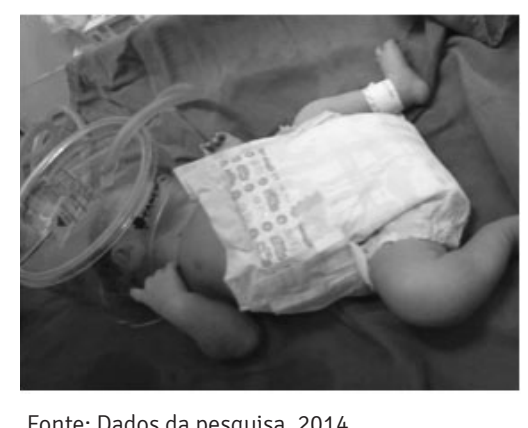

A puérpera foi recepcionada pela equipe de suporte avançado do SAMU para transferência a UTI de um hospital geral. 


\section{DISCUSSÃO}

Observou-se, no presente caso, evolução favorável da gestação abdominal, com sobrevivência da mãe e do recém-nascido. Ressalte-se, porém que esse tipo de resultado constitui a exceção, e não a regra.

De acordo com Santos e outros autores (1999), a morbimortalidade materna é elevada, sendo frequentes complicações cataclísmicas, como a hemorragia do leito placentário. Além desta, pode-se observar a infecção e, em menor frequência, a anemia, hipofibrinogenia, a obstrução intestinal, a embolia amniótica e até mesmo a formação de fístula âmnio-intestinal.

No caso aqui relatado, encontramos associação com anemia advinda da perda sanguínea materna durante o perioperatório, porém esse achado não resultou em complicações maiores para o binômio mãe-feto, visto a disponibilidade e realização de hemotransfusão.

Vale lembrar que importantes alterações clínicas cursam com o desenvolvimento da gestação extrauterina, em especial a dor abdominal, como foi referida pela gestante. Outros sintomas associados são as alterações gastrointestinais (náuseas, vômitos, constipação) e a presença de dor à movimentação fetal, que é em geral percebida nas partes altas do abdome (SANTOS, et al., 1999; NEME, 2005; HOLZHACKER, 2008)

Nesse sentido, enfatiza-se a importância da assistência pré-natal, na detecção precoce de patologias ou riscos que podem ser prevenidos, centrada no cuidado, com vistas às queixas e sentimentos apresentados pela grávida, evitando-se dessa maneira uma assistência tecnicista e biologista, onde a tecnologia de ponta seja apenas auxiliar no processo da assistência prestada.

Segundo Viellas e outros autores (2014) a assistência pré-natal é um importante componente da atenção à saúde das mulheres no período gravídico-puerperal.
Práticas realizadas rotineiramente durante essa assistência estão associadas a melhores desfechos perinatais. Segundo recomendações do Ministério da Saúde, a assistência pré-natal deve se dar por meio da incorporação de condutas acolhedoras; do desenvolvimento de ações educativas e preventivas, sem intervenções desnecessárias; da detecção precoce de patologias e de situações de risco gestacional; de estabelecimento de vínculo entre o pré-natal e o local do parto; e do fácil acesso a serviços de saúde de qualidade, desde o atendimento ambulatorial básico ao atendimento hospitalar de alto risco (BRASIL, 2006).

Com relação ao diagnóstico, o uso da ultrassonografia torna-se fundamental, em especial quando realizada em fases precoces da gestação, pois a termo 0 diagnóstico pode ser difícil mesmo para profissionais experientes. Os principais achados ecográficos característicos de gestação abdominal são a presença do feto e da placenta em localização extrauterino, partes fetais muito próximas da parede abdominal e não visualização do miométrio entre o feto e/ou a placenta e a bexiga materna. São também frequentes o oligoamnio e as apresentações anômalas (SANTOS, et al., 1999).

Almeida e outros autores (2008) afirmam que a associação da dosagem de ß-HCG com a ultrassonografia transvaginal permite detectar cerca de $90 \%$ das gestações ectópicas, qualquer que seja sua localização. A eficácia dos dois métodos associados pode chegar a $100 \%$, caso o valor do ß-HCG seja superior a $1500 \mathrm{mUI} /$ $\mathrm{ml}$ e a ecografia afirmar a ausência de saco gestacional intrauterino. Além disso, a produção desse hormônio na prenhez ectópica cresce lentamente, enquanto na gestação tópica ele dobra a cada dois dias.

Tais características tornam-se parcialmente contraditórias aos dados observados nas US realizadas durante o pré-natal e na instituição assistencial, bem como na interpretação dos dados laboratoriais e de imagem. Foram citadas observações como apresentação de feto único no compartimento intrauterino, placenta de inserção baixa, oligoamnio, apresentação córmica. 
No que diz respeito à conduta obstétrica em manter a placenta no local de inserção, são observadas várias divergências na literatura. Almeida e outros autores (2008) e Chaves Neto (2005), defendem que a placenta deve ser mantida no seu leito de implantação; a tentativa de extraí-la pode ocasionar intensa hemorragia pela rica vascularização no sítio de inserção. Em contrapartida, NEME (2005) cita que a maioria dos autores prefer extrair a placenta durante o ato operatório, pois essa atitude não agravou o prognóstico materno e a mortalidade materna foi maior quando não se retirou a placenta. As possíveis causas do aumento do índice de morbidade deixando-se a placenta no seu leito foram descritas por Mathias e Maia Filho (2000): íleo adinâmico, obstrução intestinal, peritonite e formação de abscessos que podem drenar espontaneamente para a cavidade abdominal, intestinos, bexiga ou incisão do abdome.

Deve-se enfatizar, todavia, que o aspecto relevante na evolução do presente caso foi o resultado neonatal, com extração de recém-nascido vivo, sem malformações ou deformidades, sendo raro os relatos na literatura de sobrevivência do concepto. Nesse sentido, Santos e outros autores (1999) citam a sobrevivência neonatal em cerca de $20 \%$ nas maiores séries publicadas.

\section{CONSIDERACOÕES FINAIS}

A gravidez ectópica abdominal constitui um evento extremamente raro em obstetrícia e seu diagnóstico é muito difícil, exigindo muito tirocínio dos profissionais envolvidos na assistência a gestante, ressaltando nesse âmbito o papel do enfermeiro obstetra que deveria encontrar-se na linha de frente no que concerne a assistência pré-natal.

De acordo com a Organização Panamericana de Saúde e Organização Mundial de Saúde (1977), a assistência materno-infantil deve atingir a mulher nos períodos pré-concepcional (pré-natal, parto e puer- pério), inter-concepcional, pós-concepcional. Sendo que o enfermeiro obstetra é o profissional apto para exercer as funções assistenciais, educativas, administrativas e de pesquisa.

Em decorrência de todas essas peculiaridades, julgamos relevante a publicação do caso, que foge em diversos pontos da evolução habitual de uma gravidez. Salientamos, igualmente, o êxito obtido em sua evolução, tanto do ponto de vista materno como neonatal. E enfatizamos a importância da assistência do enfermeiro obstetra durante o ciclo gravídico puerperal, não restringindo sua atuação apenas na assistência, mas também como agente multiplicador, aumentando o número de pessoas capacitadas a dar cobertura de saúde ao binômio, mãe e filho, além de não se descuidar da investigação científica, em busca de soluções para os problemas de saúde que afetam a mulher, durante a gestação, parto e puerpério.

\section{REFERÊNCIAS}

ALMEIDA, G. Gravidez Ectópica Abdominal Primária Precoce Rota: Relato de Caso. Cadernos UnifOA. 7.ed., ago. 2008.

BRASIL. Ministério da Saúde. Pré-natal e puerpério: atenção qualificada e humanizada. Manual Técnico. Brasília: Ministério da Saúde; 2006. (Série A. Normas e Manuais Técnicos; Série Direitos Sexuais e Direitos Reprodutivos - Caderno 5).

BRITO, L.R. Expressão da MUC1 nas tubas uterinas de mulheres com gravidez tubária. 2013. Dissertação (Mestrado) - Universidade Federal do Rio Grande do Sul. Faculdade de Medicina. Programa de Pós-graduação em Medicina: ciências cirúrgicas, Porto Alegre, 2013.

CHAVES NETO, H. Obstetrícia básica. São Paulo: Atheneu, 2005. 
GONZALES, M.D. Embarazo abdominal a término: reporte de um caso. Revista colombiana de obstetrícia y ginecologia, v.53, n.1, 2002, p.121-131.

HOLZHACKER, S; et al. Gravidez abdominal intraligamentar avançada: relato de caso. Revista da Associação Médica Brasileira, v.5, n.54, 2008, p.1-6.

MATHIAS, L; MAIA FILHO, N. L. Gravidez Ectópica. In: BENZERCY, R.; OLIVEIRA, H.C. (editores). Tratado de obstetrícia Febrasgo. Rio de Janeiro: Revinter, 2000, p.445-451.

MONTENEGRO, C.A.B; RESENDE FILHO, J. Obstetrícia fundamental. Rio de Janeiro: Guanabara Koogan. 10.ed., 2006, 342p.

NEME, B. Obstetrícia básica. 3.ed. São Paulo: Sarvier, 2005, 1379p.

ORGANIZACIÓN PANAMERICANA DE LA SALUD; ORGANIZACIÓN MUNDIAL DE SALUD. Comite de expertos de la OPS/OMS. Papel de la enfermeira-obstetricia em la atención materno-infantil: informe de um grupo de trabajo. Washington, D.C.; 1977, 47p. (Informe de enfermeira, 19).
SANTOS, L.C; et al. Gravidez abdominal a termo com feto vivo: Relato de Caso. Revista Brasileira de Ginecologia e Obstetrícia, v.10, n.21, 1999, p.1-9.

SILVA FILHO, M.L; MARQUES, G.S.B; NUNES, J.T. Gravidez ectópica cornual: relato de caso. Revista de Medicina e Saúde de Brasília, v.2, n.2, 2013, p.7478.

SOARES, R.C; et al. Espessura Endometrial como Fator Orientador do Tratamento Clínico da Gravidez Tubária Íntegra. Revista Brasileira de Ginecologia e Obstetrícia, v.5, n.24, 2002, p.1-10.

VIELLAS, E.F; et al. Assistência pré-natal no Brasil. Cad. Saúde Pública. Rio de Janeiro, 30 Sup: S85-S100, 2014.

YUSUF, N; et al. Advanced abdominal pregnancy with alive fetus : A case report and review. The ORION Medical Journal, v.33, n.1, 2010, p.733-734. 